\title{
The Impact of BAL Outflows on Cosmological Structure Formation
}

\author{
Nahum Arav ${ }^{1}$ \\ ${ }^{1}$ Physics Department, Virginia Tech, Blacksburg, VA 24061, USA \\ Email: arav@vt.edu
}

\begin{abstract}
Quasar feedback has been invoked as a major mechanism for influencing large-scale cosmological structure. To quantify to what extent they affect the real universe, one needs to measure the mechanical energy output of quasars and assess the ability of this energy to produce the feedback. We have developed an observational/modeling program that has yielded the first reliable kinetic luminosity determinations of quasar broad absorption line (BAL) outflows, and have demonstrated that energetically they are indeed a major contributor to AGN feedback.
\end{abstract}

Keywords. galaxies: evolution, (galaxies:) quasars: absorption lines

\section{Introduction}

The potential impact of quasar outflows on their environment has become widely recognized as agents for many AGN feedback mechanisms. To date, jets are invoked as the main feedback agent, especially for heating the intracluster medium (ICM) and inflating the large observed X-ray bubbles (e.g., McNamara et al. 2005, 2009). However, even when modeling jets with sufficient energy for feedback, a major problem arises in modeling the interaction between a narrow jet and the ICM is the coupling of the jet energy to the core regions of the ICM (Omma \& Binney 2004; Vernaleo \& Reynolds 2006, 2007; Heinz et al. 2006), thereby reducing the ability of the jet to retard cooling flows. Furthermore, due to their low mass flux and their small opening angle, narrow jets are hard-pressed to account for chemical enrichment of the ICM and intergalactic medium (IGM) and for curtailing the growth of the host galaxy.

In contrast, quasars show a more ubiquitous type of outflow $(\sim 20 \%$ of all quasars; Hewett \& Foltz 2003), where blueshifted broad absorption lines (BAL) are attributed to subrelativistic $\left(\sim 10^{4} \mathrm{~km} \mathrm{~s}^{-1}\right)$ mass ejection. In principle, these outflows should be much more efficient feedback agents than relativistic jets. The BAL outflows are wide, covering at least $20 \%$ of the solid angle, allowing for efficient ICM heating that suppresses cooling flows. They also carry thousands of times more mass flux per unit of kinetic luminosity than do relativistic jets, making them a far better agent for ICM and IGM chemical enrichment and for feedback connected with the growth of supermassive black holes.

The existence of BAL outflows has been known for more than 40 years (Lynds 1967). However, their relationship to the host galaxy and larger cosmological structures remained unknown, since their energetics and size were largely undetermined. Over the past decade, Arav's group developed a research program that overcame many obstacles in BAL outflows research and recently began to yield accurate measurements of sizes, mass flux, and kinetic luminosity for these outflows. The measured physical quantities show that BAL outflows should play a major role in AGN feedback (Arav et al. 2008; Korista et al. 2009; Moe et al. 2009; Dunn et al. 2009). 


\section{Quantifying the Contribution of BAL Outflows to AGN Feedback}

As mentioned above, subrelativistic wide outflows are in principle a better agent for feedback processes than relativistic narrow jets. The main reason outflows were not considered as important contributors to AGN feedback is the difficulty in extracting their mass flux $(\dot{M})$ and kinetic luminosity $\left(\dot{E}_{k}\right)$ from their absorption spectra. For simplicity, we assume that the outflow is in the form of a partial thin spherical shell at a distance $R$ from the central source moving radially at constant speed $v$. Under these assumptions, $\dot{M}$ is

and $\dot{E}_{k}$ is

$$
\dot{M}_{k} \sim 8 \pi \Omega R N_{\mathrm{H}} \mu m_{p} v
$$

$$
\dot{E}_{k}=\frac{1}{2} \dot{M} v^{2},
$$

where $N_{\mathrm{H}}$ is the total column density of hydrogen, $m_{p}$ is the mass of a proton, $\mu=1.4$ is the mean molecular weight of the plasma per proton, and $\Omega$ is the fraction of the shell occupied by the outflow. Spectral observations straightforwardly determine $v$. Therefore, the observational task is to measure, or constrain, $N_{\mathrm{H}}, R$ and $\Omega$. We note that equation (2.1) is a lower limit as we only detect the cold $\left(\sim 10^{4} \mathrm{~K}\right)$ BAL material. The hot X-ray phase may dominate the energetics (e.g., Costantini et al. 2007). This makes BAL outflow even more important for feedback, and also serve as motivation for hydrodynamical simulations that can probe the energy ratio between the phases.

Prior to our program, only five analyses in the literature had some handle on these two quantities, with uncertainties of 1-3 orders of magnitude (Wampler et al. 1995; de Kool et al. 2001, 2002a,b; Hamann et al. 2001). Our research group developed analysis techniques that greatly improve the accuracy in extracting $\dot{M}$ and $\dot{E}_{k}$. This is an elaborate process in which each step can produce large errors if not performed carefully. Below is a short summary of the necessary steps:

1. Measuring reliable ionic column densities $\left(N_{\text {ion }}\right)$ from the observed troughs.

2. Photoionization modeling to determine from these $N_{\text {ion }}$ the total outflowing column density $N_{\mathrm{H}}$ and ionization parameter $U$, including careful consideration of various incident ionizing continua.

3. Distance $R$ determination: (a) measuring the number density $\left(n_{\mathrm{H}}\right)$ via troughs from metastable levels and (b) using the $n_{\mathrm{H}}$ and $U$ to solve for the distance of the absorbing gas.

4. Constraining the solid angle $\Omega$ of the outflow.

5. Measuring or constraining the chemical abundances.

6. Preliminary steps. With knowledge of the type of data needed, we (a) search for appropriate targets in the SDSS quasar catalog and obtain an adequate sample and (b) observe these objects with sufficient spectral resolution and single-to-noise ratio to enable our analysis techniques.

Once these steps are completed, we can use equations (2.1) and (2.2) to extract $\dot{E}_{k}$ and $\dot{M}$, thus quantifying the magnitude of AGN feedback arising from BAL outflows.

\section{Progress to Date}

Our main result was measuring the kinetic luminosity and mass flux of several BAL outflows. The first two objects in Table 1 have a large enough kinetic luminosity $\left(\dot{E}_{k}\right)$ to play a major role in AGN feedback. Their mass fluxes are comparable to galaxies with very high star-formation rates, suggesting that we see examples of galaxy growth suppression in real time (from Moe et al. 2009; Dunn et al. 2009; Bautista et al. 2009). 
Table 1: Summary of Current BAL Outflow Results

\begin{tabular}{l|c|c|c}
\hline Object & $\begin{array}{c}\dot{M} \\
\left(M_{\odot} \mathrm{yr}^{-1}\right)\end{array}$ & $\begin{array}{c}\dot{E}_{k} \\
\left(\mathrm{ergs} \mathrm{s}^{-1}\right)\end{array}$ & $\begin{array}{c}R \\
(\mathrm{kpc})\end{array}$ \\
\hline SDSS 0838+2955 & 500 & $5 \times 10^{45}$ & 3 \\
\hline SDSS 0318-0600 & 160 & $1 \times 10^{45}$ & 6 \\
\hline QSO 2359-1241 & 20 & $3 \times 10^{43}$ & 1.3 \\
\hline
\end{tabular}

To achieve this, we made advances in the following areas:

1. Identifying and observing promising targets with high spectral resolution. Our undergraduate student Max Moe identified the best 100 follow-up targets by sifting through the 50,000 brightest SDSS quasar spectra. To date, we have acquired data, on targets from that list, using 17 observing nights on 8-m class telescopes (VLT: 12; Subaru: 2; Magellan: 2; Keck: 1) and 18 nights on 4-m class telescopes (WHT: 8; APO 3.5-m: 10).

2. Precise measurements of physical properties. We have developed techniques to measure reliable ionic column densities $N_{\text {ion }}$ for the outflows (Gabel et al. 2005, 2006; Arav et al. 2007, 2008, see $\S 3$ here), which yielded total outflowing column densities, number densities, distances from the source and temperatures to 20-30\% accuracy (Gabel et al 2006; Arav et al. 2007, Korista et al 2008). This was followed by the first reliable estimates of mass flux and kinetic luminosity (see Table 1; Moe et al. 2009; Dunn et al. 2009, Bautista et al. 2009) with an order of magnitude improvement over previous attempts (e.g., de Kool et al 2001, 2002a), as well as absolute chemical abundances in a few cases (Gabel et al 2006; Arav et al. 2007).

\section{Acknowledgements}

We acknowledge support from NSF grant AST-0507772 and from NASA LTSA grant NAG5-12867.

\section{References}

Arav, N., et al. 2007, ApJ, 658, 829

Arav, N., et al. 2008, ApJ, 681, 954

Bautista, M. A., et al. 2009, ApJ, submitted

Costatini, E., et al. 2007, A\&SA, 461, 121

de Kool, M., et al. 2002a, ApJ, 567, 58

de Kool, M., et al. 2002b, ApJ, 570, 514

Dunn, J. P., et al. 2009, ApJ, in press

Gabel, J. R., et al. 2005, ApJ, 623, 85

Gabel, J. R., et al. 2006, ApJ, 646, 742

Hamann, F. W., et al. 2001, ApJ, 550, 142

Heinz, S., Brüggen, M., Young, A., \& Levesque, E. 2006, MNRAS, 373, L65

Hewitt, P. C. \& Foltz, C. B. 2003, AJ, 125, 1784

Korista, K. T., Bautista, M. A., Arav, N., Moe, M., Costantini, E., \& Benn, C. 2008, ApJ, 688, 108

Lynds, C. R. 1967, ApJ, 147, 837

McNamara, B. R., Nulsen, P. E. J., Wise, M. W., Rafferty, D. A., Carilli, C., Sarazin, C. L., \& Blanton, E. L. 2005, Nature, 433, 45 
McNamara, B. R., Kazemzadeh, F., Rafferty, D. A., Birzan, L., Nulsen, P. E. J., Kirkpatrick, C. C., \& Wise, M. W. 2009, ApJ, 698, 594

Moe, M., et al. 2009, ApJ, 706, 525

Omma, H., Binney, J., Bryan, G., \& Slyz, A. 2004, MNRAS, 348, 1105

Vernaleo, J. C. \& Reynolds, C. S. 2007, ApJ, 671, 171

Wampler, E. J., Chugai, N. N., \& Petitjean, P. 1995, ApJ, 443, 586 